\title{
Formulation and Evaluation of Aluminum Adjuvanted Combination Vaccine with Reduced Freeze Sensitivity
}

\author{
Sujana Prasad Chittineni ${ }^{1}$, Satish Chandra Maheshwari ${ }^{2}$, Lakshmi Narasu \\ mangamoori ${ }^{3}$ \\ ${ }^{1,3}$ Department of Biotechnology, Jawaharlal Nehru Technological University, Hyderabad, India \\ ${ }^{2}$ Biological E Ltd, Hyderabad, India.
}

\begin{abstract}
Objective of present study is to formulate and evaluate freeze thaw resistant combination vaccine comprises of Diphtheria, tetanus, whole cell pertussis, hepatitis $B$ and haemophilus type $b$ conjugate vaccines. Use of surfactants, sugars, polyols and their combinations to protect vaccines from freezing effects was proved. Freeze thaw resistant combination vaccine prepared by adding novel non ionic block copolymer polaxamer. Presence of polaxamer block copolymer exerts resistance from damage caused by freezing and thawing to aluminum adjuvanted vaccine. Polaxamer 407 itself in high concentrations found suitable to reduce freeze sensitivity. Use of high concentrations of polaxamer block copolymer with concentrated formulation helps in making vaccine freeze thaw resistant up to three freeze thaw cycles. Use of high concentration of polaxamer, thermo reversible nature of polaxamer block copolymer and viscosity modification helps in heat stability improvement as well. Concentratedn formulation developed can be easily diluted or re-suspended with suitable diluent to make final vaccine with required concentration for immunization and also reduces polaxamer concentration to suitable level for human parenteral use. Polaxamer block copolymer gives advantage for easy dispersion of vaccine and making uniform suspension post re-suspension.
\end{abstract}

KEYWORDS : Freeze sensitivity, Combination vaccine, Vaccine Stability, Polaxamer, Adjuvant

\section{INTRODUCTION}

Vaccines save millions of lives, mainly children each year. With the exception of the safe water vaccination is the modality that reduces mortality rate majorly. $20 \%$ of the total born children will not get complete immunization. Many factors effecting vaccine reach to every child. $97 \%$ of the currently available vaccines require cold storage and transportation. Each year millions of doses of vaccines were spoiling due to cold chain failure. Wastage of $25 \%$ in 10 to 20 dosage forms and $10 \%$ in 2 to 6 dosage forms is projected every year due to reasons like discarding of opened vials as per multi dose vial usage policy, cold chain breakage and administration to population outside the target group. Major wastage comes from multi dose vials usage policy for multi dose vaccines and cold chain breakage. Cold chain breakage is major wastage contributor for single dose vaccines[1]. Vaccines are exposed to freezing even in tropical countries. In Malaysia: 232 out of 234 Freeze Watch ${ }^{\mathrm{TM}}$ indicators broke during storage of Hepatitis B vaccine at state stores. In Australia: $48 \%$ of Hepatitis B vaccine exposed to $<-3^{\circ} \mathrm{C}$, mostly during clinic storage. In Australia: 4 times more freezing than heat exposure (during transport and storage) happens[2].In India wastage occurs at service delivery level (27\% for DPT and $61 \%$ for BCG at outreach session site) as compared to the supply chain levels (Measles3.5\%, others $<1$ $\%)$. Poor documentation of vaccine wastage at supply chain is one of the responsible factors for this very low value. Session size, vial size, formulation (liquid vs. lyophilized, oral vs. injectable) also influences vaccine wastage. To reduce vaccine wastage with optimal increase in cold chain space and management is required[3].

Accidental freezing regularly occurs when vaccines are stored in improperly maintained, inadequate, or outdated refrigerators; exposed to ambient cold during winter transit; or, come into contact with ice or frozen ice packs during transport[4].Many vaccines including pediatric combination vaccines like DTPHHib vaccines were adjuvanted with aluminum salts (Aluminum phosphate or aluminum hydroxide). Aluminum adjuvant vaccines were sensitive to freezing and losses potency on freezing and thawing[5]. Aluminum salts were used as vaccine adjuvants from long time and process of preparation of these adjuvants is well known and commercially available for low cost. Aluminum salt adjuvants breaks down, particle size increase when subjected to freezing and thawing and also losses adsorption capacity $[6,7,8]$.The damage caused by freezing is irreversible . Freeze thawing (to $-40^{\circ} \mathrm{C}$ ) of $0.2 \%$ Alhydrogel at $\mathrm{pH} 4.0$ caused particle aggregation, which is inversely related to the cooling or thawing rate[9]. Certain processes could reverse freezing-induced aggregation of aluminum salts, such as ultrasonic treatment or homogenization, but not possible in case of vaccines filled in to vials for supply 
and antigen may be damaged[10]. Freezing of tetanus vaccine, DT vaccine and DTP vaccine made of aluminum salt adjuvant losses potency on freezing and thawing[11, 12]. Sometimes temperatures lower than $-5^{\circ} \mathrm{C}$ and multiple freeze thaw cycles is required to make vaccine ineffective. There is a possibility for multiple freezethaw cycles in vaccine cold chain maintenance $[13,14,15,16]$.

Development of formulation with certain excipients could potentially inhibit the freezing-induced particle aggregation. Aluminum adjuvanted hepatitis B vaccine prevented from agglomeration on freezing by including PEG 300, propylene glycol, or glycerol in to the formulation. High concentrations of these excipients is required for complete protection from freezing damage[17.] These excipients protect aluminum adjuvanted vaccines due to their cryo protective nature and will not reduce freezing temperature. Other excipients like adsorbable polymers or surface-active agents are also found to be effective in inhibiting freezinginduced aggregation $[18,19,20]$. High concentrations (20\%) of Cryo protective agents like propylene glycol also helps in stabilization of proteins in aluminum adjuvanted vaccines via protecting from $\mathrm{pH}$ changes on aluminum adjuvant surface due to accumulation of hydroxyl ions[21].

Liquid Diphtheria, Tetanus, Pertussis, Hepatitis B and Haemophilus type b conjugate combined vaccine (DTwP-HepB-Hib presentation) is a high-priority vaccine for WHO. This combination "five-in-one" vaccine protects children from diphtheria, pertussis (whooping cough), tetanus, hepatitis B and Haemophilus influenzae type b (Hib) which causes pneumonia and meningitis. It is less traumatic for babies to receive and easier for programmes to administer than previous formulations[22]. Four licensed Diphtheria, Tetanus, Pertussis, Hepatitis B and Haemophilus type b conjugate combined vaccines are currently prequalified by the WHO. The earlier generations of these vaccines consist of a DTPw-HepB component and a separate lyophilized Hib component that has to be reconstituted before use, while newer ones are available as fully liquid suspensions. Pack inserts of all these vaccines indicate store vaccine at a temperature between $+2{ }^{\circ} \mathrm{C}$ and $+8{ }^{\circ} \mathrm{C}$, do not freeze and discard if vaccine has been frozen. In all the four vaccines aluminum salt was used as adjuvant [23].

In this study we examined non ionic block copolymer polaxamer 407 (also called as lutrol or Pluronics (trade name of BASF)) for freeze protection activity on aluminum adjuvanted combination vaccine. Polaxamers are non toxic synthetic copolymers of ethylene oxide and propylene oxide. Polaxamers are available in pharmaceutical grade and commercially available at low cost. Polaxamers are readily soluble in water. Lutrol F 68 has been used in liquid formulation for parenteral use. Aqueous solutions containing more than $20 \%$ polaxamer 407 and 188 are thermo reversible. Repeated heating and cooling will not affect thermo reversible properties and at lower concentrations can be used to modulate viscosity of liquid formulations[24]. At high concentrations in poloxamer 407, proteins form homogeneous suspensions with full retention of native secondary structure. Pluronic block polymers were found exciting opportunities for the development of novel gene therapies and vaccination strategies[25]. Polaxamer non ionic block copolymers also exerts adjuvant activity[26, 27].

It was reported the immunostimulatory effects on the mucosal immune response of a unique adjuvant system composed of the block co-polymer, Pluronic®F127. Formulations of F127 can be administered in liquid form at temperatures less than approximately $10^{\circ} \mathrm{C}$, with conversion to semisolid gels at body temperature, thereby potentially acting as sustained release depots[ 28].

\section{1}

\section{MATERIAL AND METHODS}

Material

Diphtheria toxoid, Tetanus toxoid, Hepatitis B surface antigen, whole cell pertussis and Haemophilus type $\mathrm{b}$ conjugate were obtained from Biological E Ltd. Polxamer 407 was purchased from Sigma . Aluminum phosphate adjuvant was obtained from Brentag Biosector. AxSYM HBsAg V2 kit purchased from Abbott. Swiss Albino mice used for the pertussis potency were in-house bred in live stock division Biological E Ltd. For free PRP analysis Column MA1 was purchased from Dionex. All the chemicals used for testing were of analytical grade and purchased from commercial suppliers.

\subsection{Methods}

Stock solution of polaxamer at $40 \% \mathrm{~W} / \mathrm{V}$ prepared in cold water for injection and kept at $2-8^{\circ} \mathrm{C}$ overnight for complete dissolution. Stock solutions were sterilized by autoclaving at $121^{\circ} \mathrm{C}$ for 15 minutes and 1 bar pressure. After sterilization polaxamer stock solutions were cooled to $2-8^{\circ} \mathrm{C}$ before use[29, 30]. 


\subsection{Formulation process}

In the formulation process first Diphtheria, tetanus and hepatitis B antigens adsorbed separately to aluminum phosphate and kept for maturation for 1 week. In the container containing polaxamer solution whole cell pertussis was added and mixed thoroughly by stirring using magnetic stirrer at $150 \mathrm{rpm}$ for 4 hours. Then adsorbed diphtheria, tetanus and Hepatitis B part is transferred to the container having whole cell pertussis and polaxamer mixture, stirred for 30 minutes at $150 \mathrm{rpm}$. Then Haemophilus type b conjugate is transferred, volume was made up with normal saline and mixed for 30 minutes. During whole process temperature was maintained at $2-8^{\circ} \mathrm{C}$ and all solutions were cooled to $2-8^{\circ} \mathrm{C}$ before addition. Likewise different formulations having polaxamer concentrations of 5 to $20 \%$ were prepared and studied. $5 x$ concentrated formulations containing $250 \mathrm{Lf} / \mathrm{mL}$ of Diphtheria toxoid, $50 \mathrm{Lf} / \mathrm{mL}$ of Tetanus toxoid, $150 \mathrm{IOU} / \mathrm{mL}$ pertussis antigen, $100 \mu \mathrm{g} / \mathrm{mL}$ of hepatitis B surface antigen, $100 \mu \mathrm{g}$ per $\mathrm{mL}$ haemophilus type $\mathrm{b}$ conjugate and polaxamer 5 to $20 \%$ W/V prepared following preparation procedure defined above. $1 \mathrm{~mL}$ of formulation prepared Formulations having the 20\% polaxamer 407 was selected for the complete characterization study based on visual observation of aluminum adjuvant after freezing and thawing. Other formulations having polaxamer below $10 \%$ not exerted the freeze protection of aluminum adjuvant and agglomeration observed.

\section{$2.4 \quad$ Testing methods}

Two vaccine formulations selected were tested for different quality parameters like appearance, Shake test, hepatitis B in-vitro potency, pertussis potency, free polysaccharide for Haemophilus type b conjugate and abnormal toxicity. Considering the better stability of diphtheria, tetanus and huge animal requirement potency of these components was not studied. Both formulations were diluted to single humanizing dose level by diluting to $1 \mathrm{x}$ with water for injection. Appearance was tested visually and microscopically. Shake test was carried out as per procedure defined by WHO [31, 32, 33]. Hepatitis B in-vitro potency test was carried out using AxSYM HBsAg V2 kit from Abbott as per method defined in article published by Naresh singh gill et al.[34]. Pertussis potency was tested by intra-cerebral mice challenge assay (kendrik method)[35].

HPAEC-PAD (high performance anion-exchange chromatography with pulsed amperometric detection) method was used for free polysaccharide estimation using PRP standard obtained from NIBSC. The prepared lyophilized vaccine reconstituted with water for injection prior to free polysaccharide separation from conjugated polysaccharide. Acid hydrolysis of the standard and vaccine samples was performed using hydrochloric acid. C4 cartridge column was used for separating free from total polysaccharide prior to hydrolysis. A Dionex ion chromatography system with Dionex MA-1 column was used[36, 37].Abnormal toxicity was performed as per Indian Pharmacopoeia after 3 freeze thaw cycles[38].

\subsection{Freeze thaw studies}

Concentrated combined vaccine formulations were subjected for freeze thaw stability by incubating overnight at $-20^{\circ} \mathrm{C}$ and thawing at $2-8^{\circ} \mathrm{C}$. Likewise 3 cycles of freezing and thawing was done. After each cycle tests defined above were carried out.

\section{RESULTS}

Visually appearance of formulation with $20 \%$ polaxamer 407 post three freeze and thaw cycles was good and no agglomeration of aluminum adjuvant found. Post re-suspension appearance was good for formulation with polaxamer 20\% after three freeze thaw cycles. Microscopic examination pictures of the formulation with polaxamer and control formulation without polaxamer were shown in the Fig. 1 and 2.

Figure 1: Microscopic picture of formulation with polaxamer 20\% after 3 cycles of freeze thaw.

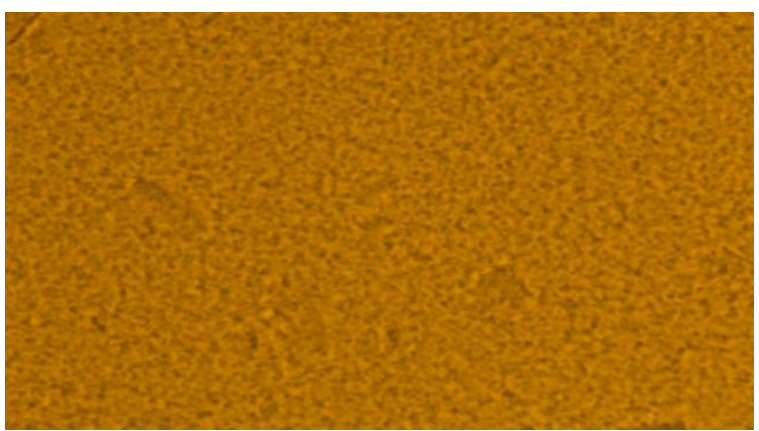


Figure 2. Microscopic picture of control formulation without polaxamer before( $2 a$ )and after single freeze thaw (2b).
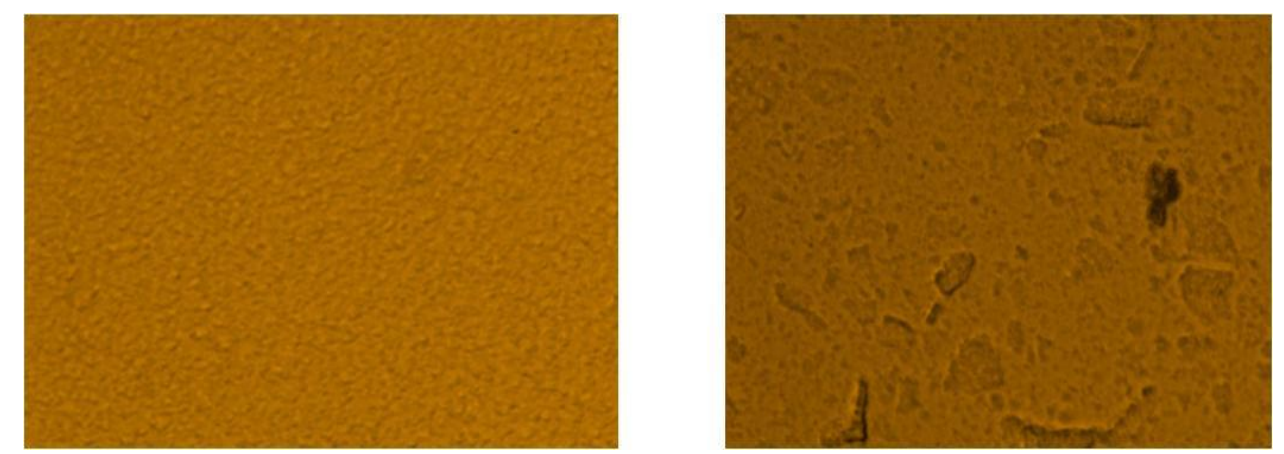

In-vitro relative potency values obtained for the formulation with and without polaxamer were tested at each freeze thaw cycle and the obtained results were shown in the Table 1.

Table 1:

\begin{tabular}{|c|c|c|c|c|}
\hline Formulation & $\begin{array}{c}\text { Before } \\
\text { freezing }\end{array}$ & $\begin{array}{c}\text { After 1st freeze } \\
\text { thaw }\end{array}$ & $\begin{array}{c}\text { After 2nd freeze } \\
\text { thaw }\end{array}$ & $\begin{array}{c}\text { After 3rd freeze } \\
\text { thaw }\end{array}$ \\
\hline With 20\% polaxamer & 1.2 & 1.2 & 1.1 & 1.1 \\
\hline Without polaxamer & 1.1 & 0.8 & 0.7 & 0.5 \\
\hline
\end{tabular}

More than $4 \mathrm{IU}$ pertussis potency was obtained for formulation with polaxamer after exposure to three freeze thaw cycles when tested in mice by intra cerebral challenge method and complied with requirements [35, 38]: Pertussis potency results were shown in Table 2

Table 2:

\begin{tabular}{|l|c|l|}
\hline Formulation & Before freezing & After three freeze thaws \\
\hline With 20\% polaxamer & $6.8 \mathrm{IU} /$ dose & $6.4 \mathrm{IU} /$ dose \\
\hline Without polaxamer & $5.6 \mathrm{IU} /$ dose & $3.2 \mathrm{IU} /$ dose \\
\hline
\end{tabular}

Estimated free PRP (unconjugated polysaccharide) was less than $20 \%$ in formulation with polaxamer when tested using HPAEC-PAD method after freezing and thawing $[38,39]$. Obtained free PRP Results at each freeze thaw cycle were shown in table 3.

Table 3:

\begin{tabular}{|c|c|c|c|c|}
\hline Formulation & Before freezing & After 1st freeze thaw & After 2nd freeze thaw & After 3rd freeze thaw \\
\hline With 20\% polaxamer & $8 \%$ & $8.4 \%$ & $9 \%$ & $10.2 \%$ \\
\hline Without polaxamer & $9.6 \%$ & $14 \%$ & $22 \%$ & $26 \%$ \\
\hline
\end{tabular}


Figure 3: Chromatogram showing free PRP peak in HPAEC-PAD analysis.

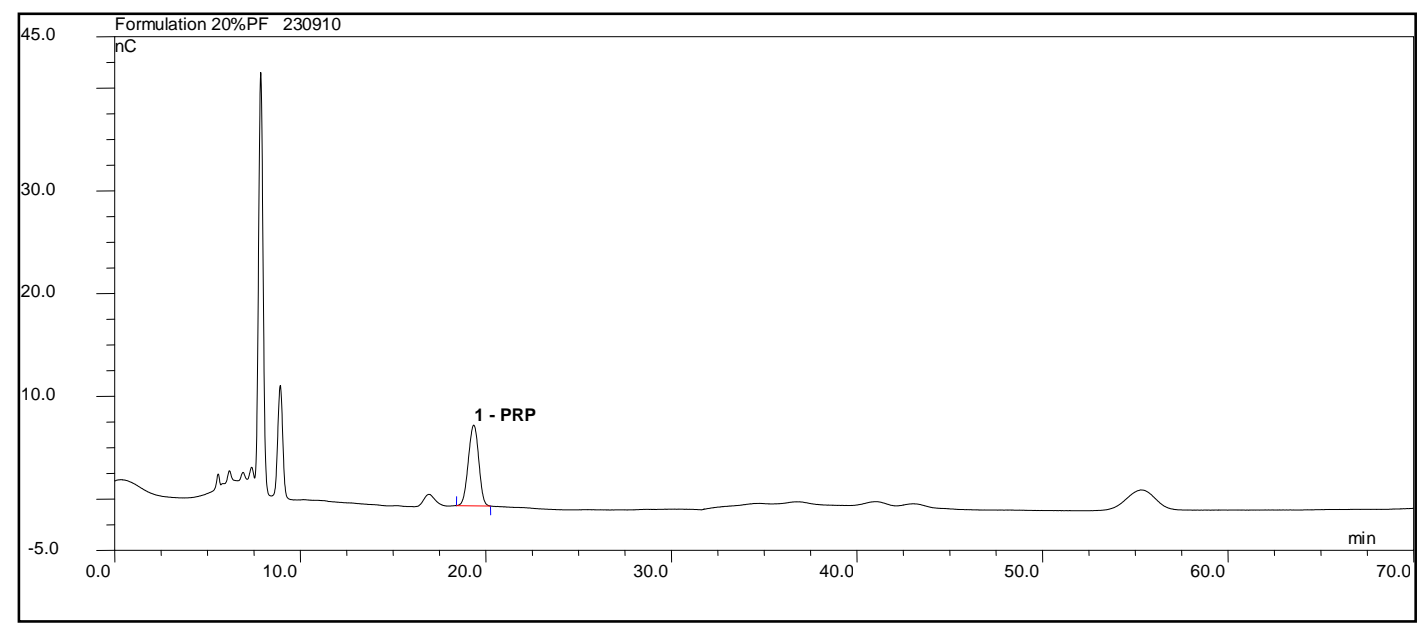

Shake test pictures of Formulation with $20 \%$ polaxamer were shown in figure 4 . sedimentation time for formulation with $20 \%$ polaxamer is similar to unfrozen vaccine formulation. Formulation with $20 \%$ polaxamer passed the Abnormal toxicity requirements as per Indian Pharmacopoeia[38] after 3 freeze thaw cycles.

Figure 4: After standing for 10 minutes formulation with 20\% polaxamer after 3 freeze thaw cycles (Left), Control formulation without polaxamer after one freeze thaw cycle (right)

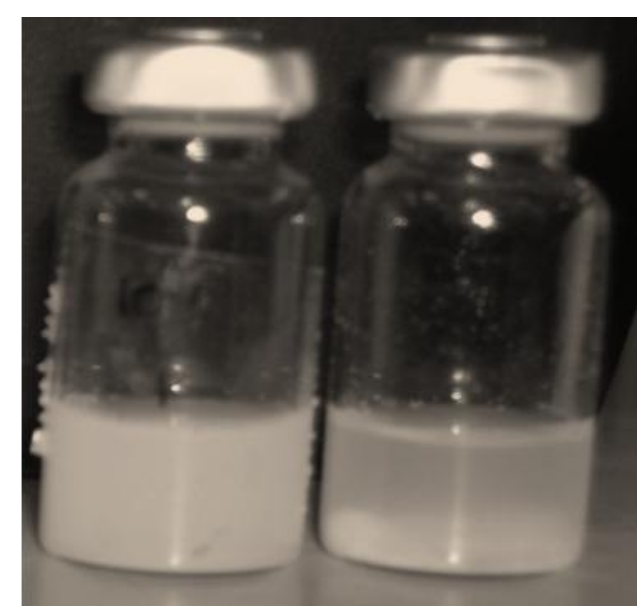

\section{DISCUSSION}

Results indicates that use of $20 \%$ polaxamer can protect Diphtheria, Tetanus, Pertussis, Hepatitis B and Haemophilus type $b$ conjugate combined vaccine from at least 3 cycles of freezing and thawing. Microscopic images (Fig. 1,2 ) clearly show no damage to aluminum adjuvant in vaccine formulation with $20 \%$ polaxamer, at the same time normal formulation without polaxamer protective agent showed agglomeration of aluminum adjuvant. When subjected to freezing no loss of hepatitis B in-vitro potency observed in formulation having $20 \%$ polaxamer and about $50 \%$ loss in potency was observed in formulation without polaxamer protective agent (Table 1). In case of pertussis potency minimal reduction was observed in formulation with polaxamer on exposure to freezing (Table 2). Minimal increase in \% free PRP was observed in formulation having $20 \%$ polaxamer where as free PRP crossed $20 \%$ in formulation without polaxamer protective agent (Table 3 ). No increase in sedimentation time was observed in formulation with $20 \%$ polaxamer when subjected to 3 freeze thaw cycles, in formulation without polaxamer protective agent clear sediment was observed after standing for 10 minutes (Fig.4). 


\section{CONCLUSIONS}

Diphtheria, Tetanus, Pertussis, Hepatitis B and Haemophilus type b conjugate combined vaccine was successfully protected from minimum three freeze thaw cycles with use of protective agent in formulation. Nonionic block copolymers polaxamer 407 found suitable as protective agent against freezing effects on aluminum adjuvant vaccines. High concentration $(20 \%)$ of polaxamer is required to protect from freezing. Concentrated formulation with high concentrations of protective agent for extemporaneous re-dilution or resuspension found suitable option to make freeze resistant vaccine. Prepared concentrated Diphtheria, Tetanus, Pertussis, Hepatitis B and Haemophilus type b conjugate combined vaccine formulation was easily re-suspend able using water for injection before immunization.

\section{ACKNOWLEDGEMENT}

The authors thank Biological E Ltd for the providing antigens and the assistance for the analytics.

\section{REFERENCES}

[1]. Safe vaccine handling, cold chain and Immunizations, a manual for the Newly Independent States, WHO/EPI/LHIS/98.02

[2]. The clear and pressing need for a reliable method for detecting and preventing the use of freeze-damaged biopharmaceuticals, Milan Innovation Limited - February 2011.

[3]. Unicef, Vaccine Wastage Assessment, Field assessment and observations from National stores and five selected states of India April 2010.

[4]. Debra Kristensen, Dexiang Chen, Ray Cummings, Vaccine stabilization: Research, commercialization, and potential impact, Vaccine, 2011, 29, 7122- 7124.

[5]. Maa YF, Zhao L, Payne LG, Chen D. Stabilization of alum-adjuvanted vaccine dry powder formulations: mechanism and application. J Pharm Sci 2003, 92, 2, 319-32.

[6]. US PATENT 4578270, 1986

[7]. Chen D, Tyagi A, Carpenter J, Perkins S, Sylvester D, Guy M, Characterization of the freeze sensitivity of a hepatitis B vaccine. Hum Vaccines 2009, 5, 1:1-7.

[8]. Clausi A, Cummiskey J, Merkley S, Carpenter JF, Braun LJ, Randolph TW. Influence of particle size and antigen binding on effectiveness of aluminum salt adjuvants in a model lysozyme vaccine. J Pharm Sci 2008, April 8.

[9]. Wei Wang1, Manmohan Singh2, Selection of Adjuvants for Enhanced Vaccine Potency, World Journal of Vaccines, 2011, 1, 3378.

[10]. M. I. Zapata, J. R. Feldkamp, G. E. Peck, J. L. White and S. L. Hem, "Mechanism of Freeze-Thaw Instability of Aluminum Hydroxycarbonate and Magnesium Hydroxide Gels," Journal of Pharmaceutical Sciences, Vol. 73, 1984,1, 3-8.

[11]. Dietz V, Galazka A, van Loon F, Cochi S. Factors affecting the immunogenicity and potency of tetanus toxoid: implications for the elimination of neonatal and non-neonatal tetanus as public health problems. Bull World Health Organ 1997;75:81-93.

[12]. The effects of freezing on the appearance, potency, and toxicity of adsorbed and unadsorbed DPT vaccines. Weekly Epidemiological Record 1980;55:385-92.

[13]. Nelson C, Froes P, Dyck AM, Chavarria J, Boda E, Coca A, Monitoring temperatures in the vaccine cold chain in Bolivia. Vaccine 2007, 25, 3, 433-7, 8 .

[14]. Techathawat S, Varinsathien P, Rasdjarmrearnsook A, Tharmaphornpilas P. Exposure to heat and freezing in the vaccine cold chain in Thailand. Vaccine 2007, 25, 7, 1328-33.

[15]. Wirkas T, Toikilik S, Miller N, Morgan C, Clements CJ. A vaccine cold chain freezing study in PNG highlights technology needs for hot climate countries. Vaccine 2007, 25, 4, 691-7.,9.

[16]. Dimayuga R, Scheifele D, Bell A. Effects of freezing on DPT and DPT-IPV vaccines, adsorbed. Can Commun Dis Rep, 1995, 21:101-3.

[17]. D. Chen, A. Tyagi, J. Carpenter, S. Perkins, D. Sylvester, M. Guy, D. D. Kristensen and L. J. Braun, "Characterization of the Freeze Sensitivity of a Hepatitis B Vaccine," Human Vaccines, 2009, 5, 1, 26-32.

[18]. M. I. Zapata, J. R. Feldkamp, G. E. Peck, J. L. White and S. L. Hem, "Mechanism of Freeze-Thaw Instability of Aluminum Hydroxycarbonate and Magnesium Hydroxide Gels," Journal of Pharmaceutical Sciences, 1984,73, 1, 3-8.

[19]. L. J. Braun, A. Tyagi, S. Perkins, J. Carpenter, D. Sylvester, M. Guy, D. Kristensen and D. Chen, "Development of a FreezeStable Formulation for Vaccines Containing Aluminum Salt Adjuvants," Vaccine, 2009, 27, 1, 72-79.

[20]. A. L. Clausi, A. Morin, J. F. Carpenter and T. W. Randolph, "Influence of Protein Conformation and Adjuvant Aggregation on the Effectiveness of Aluminum Hydroxide Adjuvant in a Model Alkaline Phosphatase Vaccine," Journal of Pharmaceutical Sciences, 2009, 98, 1, 114-121.

[21]. L. J. Braun, J. Jezek, S. Peterson, A. Tyagi, S. Perkins, D. Sylvester, M. Guy, M. Lal, S. Priddy, H. Plzak, D. Kristensen and D. Chen, "Characterization of a Thermostable Hepatitis B Vaccine Formulation," Vaccine, 2009, 27, 34, 4609-4614.

[22]. WHO, Vaccines prequalification priority list 2013-14.

[23]. WHO, List of prequalified vaccines for supply to UN agencies.

[24]. Polaxamers for pharmaceutical use, Technical information from BASF, April 2010.

[25]. Alexander V. Kabanov, Elena V. Batrakova, Valery Yu. Alakhov, Pluronic block copolymers as novel polymer therapeutics for drug and gene delivery Journal of Controlled Release, 2002, 82, 189-212.

[26]. Polaxamer non ionic block copolymers also exerts adjuvant activity, A.C. Allison, N.E. Byars, Adjuvant formulations and their mode of action, Semin. Immunol. 1990, 2, 369-374. 
[27]. M.J. Newman, J.K. Actor, M. Balusubramanian, C. Jagannath, Use of nonionic block copolymers in vaccines and therapeutics, Crit. Rev. Ther. Drug Carrier Syst. 1998, 15, 89-142.

[28]. Claire M. Coeshott, S. Louise Smithson, Evie Verderber, Adrian Samaniego, Joan M. Blonder, Gary J. Rosenthal, M.A. Julie Westerink, Pluronic® F127-based systemic vaccine delivery systems, Vaccine, 2004, 22, 2396-2405.

[29]. Mark S. F. Clarke and Paul L. McNeil, Syringe loading introduces macromolecules into living mammalian cell cytosol, Journal of Cell Science, 1992, 102, 533-541.

[30]. B. Fussnegger, LutrolY F 127 (Poloxamer 407), BASF ExAct, April, 2000, 4, page 7.

[31]. Umit Kartoglu, Nejat Kenan Ozgüler, Lara J Wolfson,Wiesław Kurzatkowski, Validation of the shake test for detecting freeze damage to adsorbed vaccines, Bulletin of the World Health Organization 2010;88:624-631

[32]. Pan american health organization immunization newsletter Volume XXXII, Number 2 April 2010

[33]. WHO learning guide on how to use the shake test, go to https://apps.who. int/vaccines-access/vacman/temperature/ shake_test_learning_guide.html.

[34]. Naresh Singh Gill, Poonam Kumari, AxSYM Qualitative and quantitative analysis of various constituents of vaccines by using analytical techniques, Pharmacophore 2011, Vol. 2 (3), 186-194.

[35]. Recommendations for whole-cell pertussis vaccine. Annex 6 in: WHO Expert Committee on Biological Standardization. Fiftysixth report. Geneva, World Health Organization, 2007 (WHO Technical Report Series, No. 941).

[36]. Angela Bardotti, Neil Ravenscroft, Stefano Ricci, Sandro D'Ascenzi, Valentina Guarnieri, Giovanni Averani, Paolo Constantino, Quantitative determination of saccharide in Haemophilus influenzae type b glycoconjugate vaccines, alone and in combination with DPT, by use of high-performance anion-exchange chromatography with pulsed amperometric detection Vaccine 2000, 18(19): 1982-1993.

[37]. Lokesh Bhattacharyya, Jeffrey S. Rohrer, Applications of Ion Chromatography in the Analysis of Pharmaceutical and Biological Products, John Wiley \& Sons, 10-Feb-2012.

[38]. Indian Pharmacopoeia 2010.

[39]. Recommendations for the production and control of Haemophilus influenzae type b conjugate vaccines, Annex 1 in: WHO Expert Committee on Biological Standardization. Forty-ninth report. Geneva, World Health Organization, 2000 (WHO Technical Report Series, No. 897). 